\title{
An Improved Optimal Path and Energy Efficiency Routing Algorithm in Cyber-Physical Systems for Real-Time Basketball Player Monitoring
}

\author{
Shiliang Luo ${ }^{1,2}$, Lianglun Cheng ${ }^{1}$ and Bin $\operatorname{Ren}^{3}$ \\ ${ }^{1}$ School of Automation, Guangdong University of Technology, Guangzhou, China \\ ${ }^{2}$ School of Mathematics \& Computer Science, Gannan Normal University, Ganzhou, \\ China \\ ${ }^{3}$ School of Electronic Engineering, Dongguan University of Technology, Dongguan, \\ China \\ luoshiliang88@163.com ${ }^{1,2}$,llcheng@gdut.edu.cn ${ }^{1}$, reb100@163.com ${ }^{3}$
}

\begin{abstract}
Live physiological monitoring of basketball players during events can help maximize athlete performance while preventing injury and enhance TV broadcast services. However, the harsh conditions in the basketball court pose many challenges. In this paper, we propose an improved optimal path and energy efficiency sensor routing algorithm for athletes' monitoring services. The goal is to pave the way to the realisation of real-time athlete monitoring systems. In our scheme, the basketball court diagram and player default positions are given for the connectivity data collection. Then the radio signal propagation model is set up. Additionally multi-hop routing algorithms for real-time monitoring are analysed. Finally the IOPEESRA algorithm is proposed. The simulation results show that our proposed algorithm has a good performance in terms of average energy comsumption compared with the algorithm of M-LEACH and ARPEES.
\end{abstract}

Keywords: Athlete Monitoring; Process Control; Automation; Wireless Sensor Networks; Cyber-Physical Systems (CPS)

\section{Introduction}

Basketball is a popular sport throughout the world, and attracts large financial investment, particularly in USA. Many basketball clubs have expressed great interest in monitoring their athletes on the field, predominantly to reduce the risk of injury and improve player substitution decisions $[1,2]$. Basketball association has also expressed interest in using realtime position and impact information for referee-assist services. And television channels are eager to augment live broadcasts with player parameters in order to heighten the level of engagement for audiences [3]. The player parameters are impact levels during collisions, acceleration, speed, heart-rate, etc.

At present, medical technology has been used by professional coaches in striving to push their athletes' bodies to the edge of its abilities $[4,5]$. However, much of this examination of the body has been done under lab conditions where results achieved in the artificial environment may not parallel those observed in competition $[6,7]$. Now devices are starting to emerge in the market which are able to monitor athletes in training $[8,9]$. 
In the meantime, advances in communication and sensing technologies are enabling new low-cost and lightweight devices that allow measurement and remote monitoring of an individual's vital physiological signs $[10,11]$. Such technology is being adapted to the popular and growing field of sports science, especically for the athlete monitoring [12].

While hardware platforms for athlete monitoring are immature, there is much research needed in developing communication protocols that can operate in the basketball court: (a) Monitoring devices for basketball are expected to have extremely limited battery power and restricted radio range constraints on the communication protocols [13]. (b) Basketball players move rapidly in the field, and it makes the topology highly dynamic. It is challenging to design routing mechanisms that can deliver data to the base stations within stringent time and energy constraints in the time-varying environment [14]. (c) Rapid acceleration and impact are part of the sport, and it restricts the monitoring device to be small and lightweight so that the players' degree of freedom is not limited [15]. (d) The playing area in basketball is relatively large. And the transmission range of body-worn devices is limited. Real-time extraction of player data would be required.

To the best of our knowledge there is little work in mornitoring the operating environment for a mobile body-worn wireless sensor network in a basketball court. In this paper we do experimental work by sensor devices and collect data on their movement and connectivity over multiple games.

The rest of the paper is organized as follows: Section 2 illustrates the connectivity data collection. Radio signal propagation modelling is described in Section 3. Multi-hop routing algorithms for real-time monitoring are described in Section 4. Results and discussion are given in Section 5. We conclude the paper in Section 6.

\section{Connectivity Data Collection}

The game was played on a full size court with dimensions $28 \mathrm{~m} \times 15 \mathrm{~m}$. Each of the 5 players wore a monitoring device on their arm and 8 base-stations were positioned. The base-stations used the standard quarter-wavelength antenna. Figure 1 shows the nominal playing positions and associated node identification numbers.

The body-worn devices we used are off-the-shelf devices operating in the $2.4 \mathrm{GHz}$ band which are readily available today. Though they were not designed for body-worn applications, they have been used for body health monitoring, such as in Harvard's Code Blue project [14].

Taking into consideration aspects such as attenuation of the wireless signal by the body, ease and stability of attachment, and possibility of damage to the device itself, we decided to go with an arm mounted attachment using an arm-band. We implemented software on each of the body-worn devices such that it broadcasted at the highest available power level of $2 \mathrm{~mW}$. A packet containing its unique identifier and a sequence number. The devices which successfully receive this packet record this event in their onboard memory. As the game proceeds, each node will be catalogued which nodes it could hear at each time instant. In order to prevent collisions, each second is divided into 10 slots, and each of the 10 bodyworn devices is given a unique slot for transmission every second. The master base-station sends a clock synchronisation message to all nodes, on receipt of which each node starts recording connectivity data in on-board memory. Considering the limitation on the on-board space for storing the connectivity data, data collection stops after 30 minutes. And data from each node is extracted by the master base-station for off-line analysis at the end of the game. 


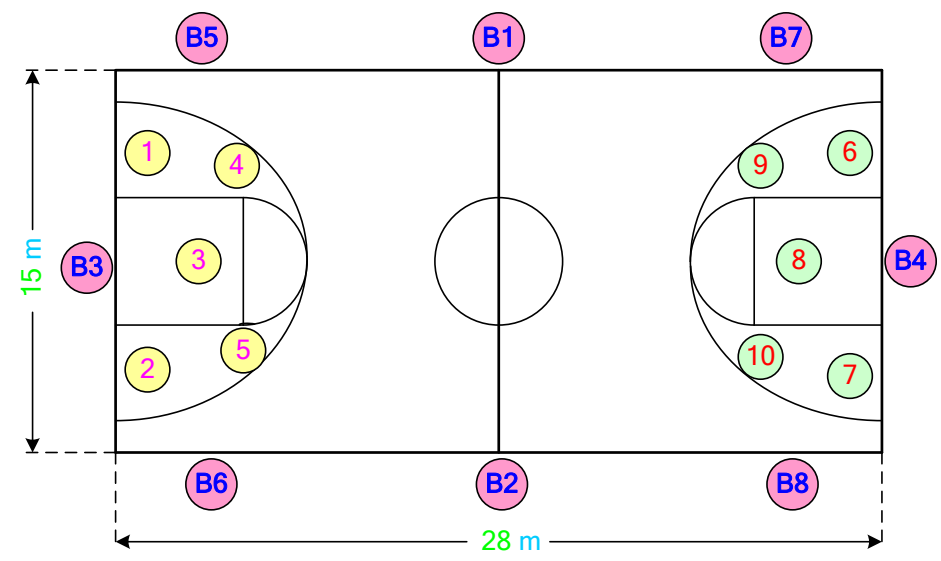

Figure 1. Basketball Court Diagram and Player Default Positions

\section{Radio Signal Propagation Modelling}

The objective in this section is to develop a model of radio signal propagation. During the game the sensor device worn by the basketball player can be used to deduce interconnectivity amongst basketball players based on their orientation and location. In our experiments we observed that the signal strength for body-worn devices is greatly infiuenced by body orientation because of the absorption by the body. There has been some prior work in evaluating the infiuence of the human body on wireless signal propagation characteristics. For example, [16] studies the effect of the human bodyon WiFi propagation from portable computers, and shows that there is a $25 \mathrm{~dB}$ loss in signal strength when the human body is in the way. The propagation of radio signals in and around the body has been studied extensively by Hall and Yao [17]. Their study concentrates mostly on on-body networks, where the transmitter and receiver are both on the same body, and shows that absorption due to the water content of the human body resulted in a $40 \mathrm{~dB}$ path loss. We have not been able to find a characterisation in the literature of radio signal strengths between a body-mounted transmitter and an off-body receiver, and in what follows we develop such a model based on empirical data, building on our recent work in [18].

As the transmitter represented the body-worn sensor device, it used a $1 / 4$ wave length dipole antenna that comes standard with the Micamotes. The receiver represented a base station, and therefore used a bigger high-gain $(+12 \mathrm{dBi})$ antenna fromTP-Link [19]. Our objective was to characterise how the received signal strength varied with the relative position between the transmitter and receiver. The transmitter sent packets at a fixed rate of eight packets per second, at a fixed power level of $2 \mathrm{~mW}$. Upon successfully receiving a packet, the receiver computed the Received Signal Strength Indicator (RSSI) of the received packet and sent this value to a laptop computer over the serial port. The RSSI value is obtained by sampling the on-board ADC during the packet reception. The RSSI value was then converted to a $\mathrm{dBm}$ value by subtracting 45 [20]. Our experiments were performed in an open basketball court away from any sources of interference.

Using the empirical data, we now derive an analytical model to deduce the signal strength as a function of distance and the orientation of the receiver from the transmitter. Free-space models typically set the received signal strength to fall with distance $r$ as a power-law.

$$
R S S I=a * r^{-b}
$$


Where a is a proportionality constant incorporating effects of the environmental variables, antenna, and transmit power. $\mathrm{b}$ is close to 2.5 .

We keep our formulation consistent with this power-law. Hence the RSSI for the bodyworn scenario takes the following expression.

$$
R S S I=a_{\rho} * r^{-b_{\rho}}
$$

The best-fit values of $a_{\rho}$ and $b_{\rho}$ for the various values of $\rho$ obtained from the experimental data at $30^{\circ}$ increments. We evaluated the fitting method on the goodness of the fit and the basis of simplicity of the expression.

It was found that the exponent $a_{\rho}$ was fitted best by a second order Fourier series of the following expression.

$$
-\log a_{\rho}-7.87=0.54 \sin (\rho * 1.98)+0.19 \cos (\rho * 1.98)-0.18 \sin (\rho * 0.99)-1.56 \cos (\rho * 0.99)
$$

The exponent $b_{\rho}$ was fitted best by a second order Fourier series of the following expression.

$$
-\log b_{\rho}-1.3=-0.3 \sin (\rho * 1.94)-0.14 \cos (\rho * 1.94)+0.19 \sin (\rho * 0.97)+0.81 \cos (\rho * 0.97)
$$

\section{Multi-Hop Routing Algorithms for Real-Time Monitoring}

We evaluate routing mechanisms for extraction of player data in this section. Then we develop a practical and efficient routing mechanism that achieves the desired trade-off between energy performance and data delivery delay.

\subsection{Multi-Hop Routing}

It is showed that multi-hop routing has the potential to significantly reduce delays in extracting data from players in the basketball court. We assume that the player-worn devices generate a sample every 2 seconds, and that every device is given one transmission opportunity. There are two schemes in delivering a sample from an athlete-worn device to the base-stations. One is flooding delivery. The other is direct delivery. The difference between them is the mode of data transmission. For the former, data from a player is forwarded by all recipients, and information propagates by one hop in one second till it reaches a base-station. Since all routing paths to base-stations are tried simultaneously, flooding is delay-optimal. While resources are expensive. For the latter, the player's tranmission is received directly by a base-station. Since it does not require store-and-forward routing, direct delivery has the lowest overheads. While it is expected to incur high delay. Flooding delivery is the lower bound and direct delivery is the upper bound on the achievable delay.

\subsection{Routing Protocol Requirements}

Direct delivery gives an upper limit while flooding gives a lower limit on delivery delays. While in terms of energy, direct delivery is much more efficient than flooding. It is because direct delivery requires fewer transmissions compared to flooding. It is indicated that flooding 
can reduce data extraction delays at the cost of increasing energy consumption. In what follows, we develop a new multi-hop routing mechanism that allows the trade-off between energy and delay.

\subsubsection{Replication at the Source}

In the proposed scheme, a player maintains a window of samples of length $\mathrm{W}$ for itself in a FIFO manner. So a newly generated sample pushes out the oldest sample in the window. The player transmits its entire window of samples during its slot. Thus every sample is duplicated W times by the source. This guards against the case where a sample may be lost because no other player is able to hear the transmitter at that time.

\subsubsection{Duplication at Intermediate Nodes}

Every player maintains a window of $\mathrm{W}$ samples for every other player. In fact it allows duplication of the samples at every intermediate hop. When a player is not transmitting, it listens to other players transmisions. Next packets heard from other players are analysed for data. If a received packet contains a more recent window of samples for player $i$, this data replaces the contents of the window for player $i$ held by player $\mathrm{j}$.

\subsubsection{Data Freshness}

The above two steps limit the amount of data transmitted in any packet. However, we still have the issue of old data being forwarded within the network. When player $j$ forwards its data to player $\mathrm{i}$, and becomes disconnected from other players. The data from player $\mathrm{j}$ may be obsolete in this case, yet it continues to be forwarded by player $i$ and its neighbours.

In order to ensure data freshness, player $i$ forwards data for player $j$ only if the most recent sample in its window for player $\mathrm{j}$ is less than $\mathrm{T}$ samples old. So each intermediate node filters the data that it receives from surrounding players before forwarding it on. The quantity $\mathrm{T}$ can be selected based on the requirements of the application. For example, for television broadcast enhancement where player heart rate is streamed in real time, samples which are more than 8 seconds old may be irrelevant, and $\mathrm{T}$ should be set to 8 .

\subsection{Operation of the Multi-Hop Routing}

Our flooding-based scheme will actively drop a window of samples as their age beyond $\mathrm{T}$. As a result, certain samples may not be delivered to the base. Thus we need to evaluate an additional parameter, the delivery ratio of this scheme.

Note that $\mathrm{W}$ controls the amount of duplication at the source, while $\mathrm{T}$ controls the amount of duplication in the network. A smaller value of T means that a given window of samples is likely forwarded through a smaller number of hops. Hence small value of $\mathrm{T}$ results in lower delivery ratios. 
Finally, the maximum time a sample can spend in the network is $\mathrm{W}+\mathrm{T}$. we have chosen to implement our algorithm in this form rather than in terms of hop counts for the following two reasons:

(1) Using a window of samples for each player allows us to naturally control another important parameter, the memory requirements of this scheme.

(2) If every player maintains a buffer of $\mathrm{W}$ samples for every other player, it is artificial to impose a hop count on every sample in that buffer. Rather, it is simpler to impose a maximum age $\mathrm{T}$ on the buffer, and to discard the entire buffer if the data is too old.

We assume that the base can inform a player when its transmission is received, on which the player erases its entire window of samples.

\section{Improved Optimal Path and Energy Efficiency Sensor Routing Algorithm}

Innitially, the cluster head broadcasts request-relay message that contains the the cluster head identification ( ID-ch), the distance d(BS,CH) from the base station to the cluster head. The last parameter flag is the acknowledgement message required conde with the default value 1 . All the nodes that receive the request-relay message will calculate the $\cos a_{i}$ with $a_{i}$ is the angle value between the cluster head, the base station and node $i$. The $\cos a_{i}$ is calculated as follows:

$$
\cos a_{i}=\frac{d^{2}(B S, C H)+d^{2}(i, C H)-d^{2}(B S, i)}{2 d(B S, C H) \cdot d(i, C H)}
$$

When the value of flag is 1 , the node that has the value $\cos a_{i} \geq 0$ needs to reply by sending ACK-relay message. This will reduce the number of control message significantly, hence prolonging the lifetime of the network. If there are not a node that satisfies the condition after the time period, the cluster head resends the request-relay message with the new flag value. In this case, all the node that receive the request-relay message have to reply the cluster head by sending the ACK-relay message. The cluster head will calculate the RELAY-node function after receiving the ACK-relay messages.

$$
F(i)=\frac{d(B S, i)}{d(C H, i)}+\sin a_{i}+\frac{q}{E(i)}
$$

The node with the minimum RELAY-node function is choosed to be the relay node. The meaning of RELAY-node function is the estimated cost of the path from the cluster head to the base station passing the intermediate node $i$. the node which owns the minimum value cost is selected as the relay node. The weight $\mathrm{q}$ is used to adjust the importance of residual energy on RELAY-node function. Figure 2 shows the flowchart of the IOPEESRA. 


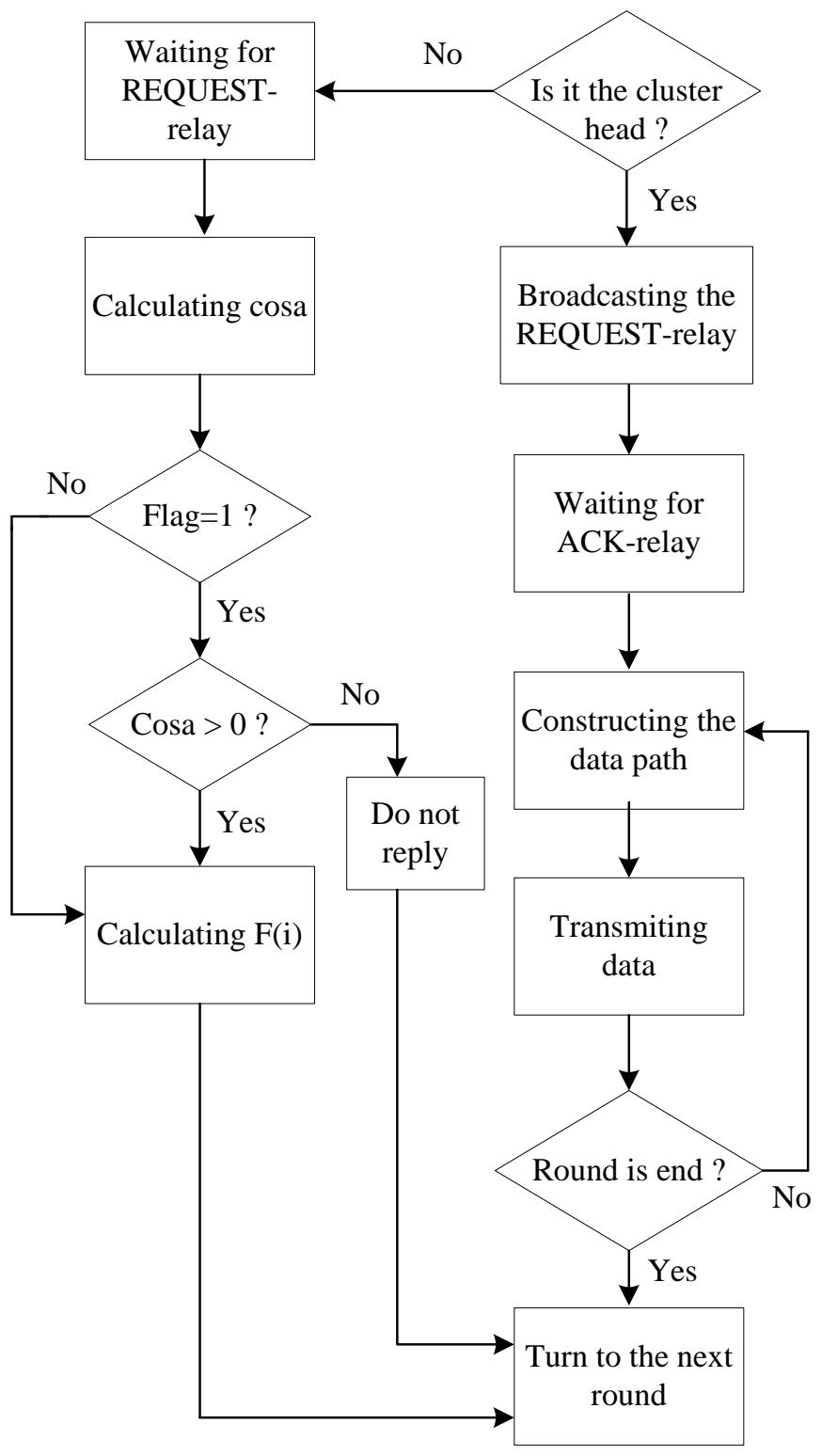

Figure 2. Flowchart of the IOPEESRA

\section{Results and Discussion}

In this paper, we compared the DV-Hop algorithm and the improved algorithm. In order to test the performance of the improved algorithm, we did lots of experiments with the simulation tools OMNeT++ simulator and MATLAB.

\subsection{Simulation Parameter Settings}

The node sensing range is $75 \mathrm{~m}$. The transmission range is $100 \mathrm{~m}$. The initial energy is 2 Joule. Broadcast packet size is 25 byte. Data packet size is 500 byte. Netwotk dimension is $28 \mathrm{~m} \times 15 \mathrm{~m}$. 


\subsection{Ttotal Network Residual Energy After the Death of One Third Nodes}

Figure 3 shows that the residual energy of IOPEESRA is always better than M-LEACH and ARPEES. After 150 rounds, the energy of M-LEACH and ARPEES is approximately 150(J), while IOPEESRA achieves about 170(J). Additionally, the lifetime of M-LEACH and ARPEES is only 200 rounds, while IOPEESRA's lifetime is 400 rounds. It is indicated that our proposed IOPEESRA scheme can prolong the network lifetime. The reason is that, the number of broadcasting messages is greatly reduced by IOPEESRA, and thus the network lifetime is obviously proloonged.

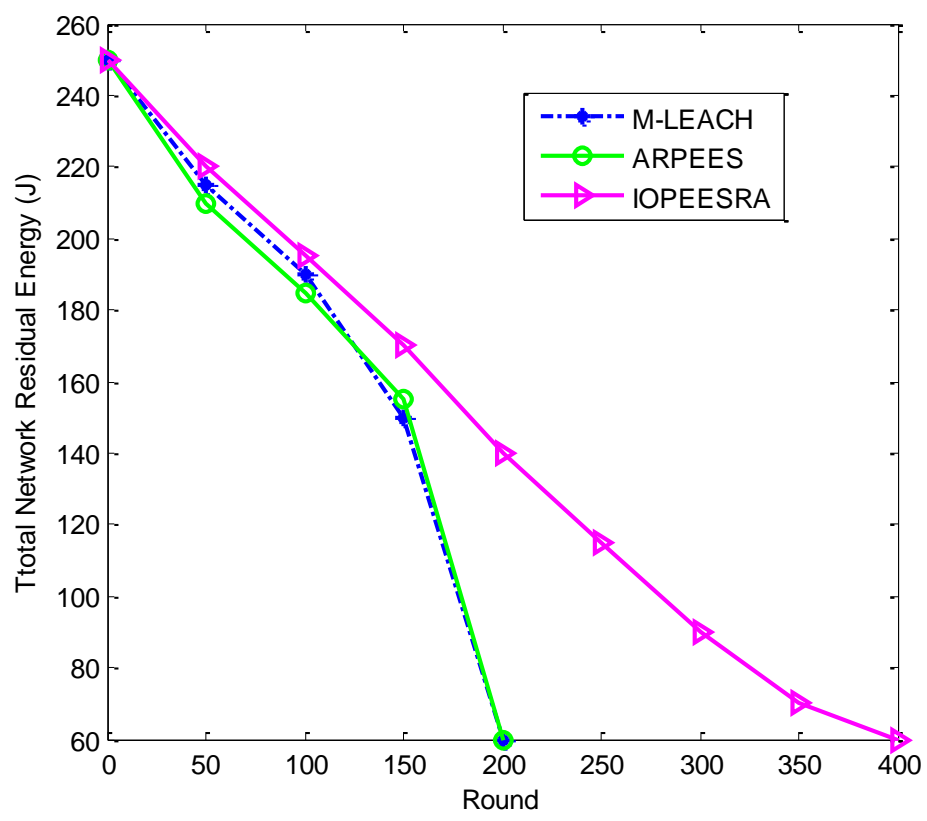

Figure 3. Ttotal Network Residual Energy

\subsection{Number of Aliving Node After 180 Rounds}

Figure 4 shows that the number of aliving node of M-LEACH and ARPEES decrease significantly. The number of aliving node in IOPEESRA stays larger and more stable than two others. It is also indicated that our proposed IOPEESRA scheme outperforms the MLEACH and ARPEES. The reason is that, the RELAY-node function chooses an optimal algorithm which is based on the residual energy, the distance and the angle value. Compared to the M-LEACH and ARPEES, the path of approaching the base station in IOPEESRA is more straight and shorter. So the consumed energy in IOPEESRA is obviously reduced. And the number of aliving node in IOPEESRA is more than that in M-LEACH and ARPEES. 


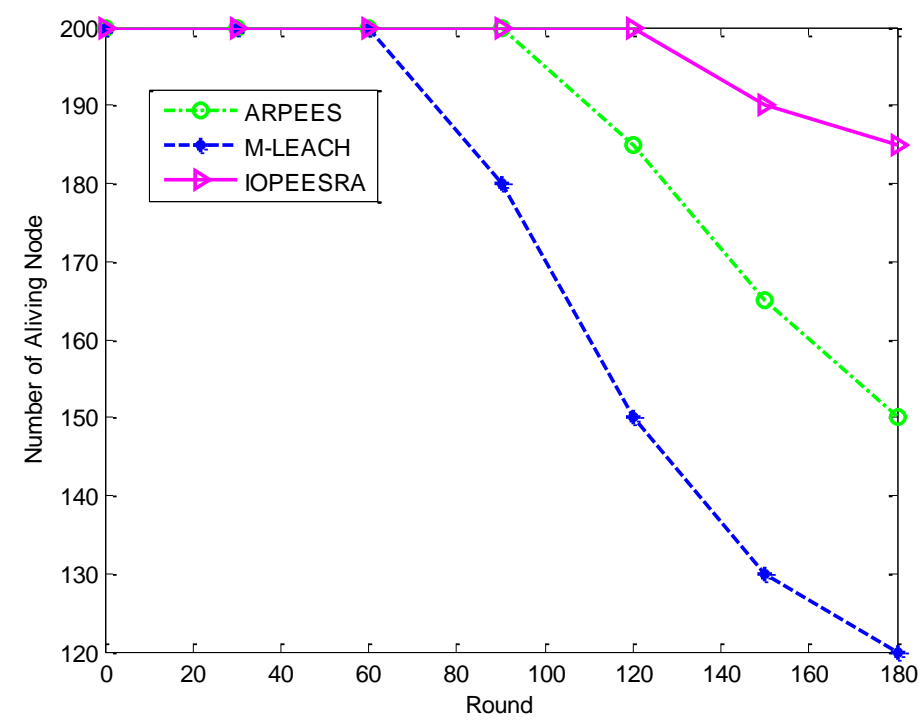

Figure 4. Number of Aliving Node

\subsection{Ratio of Clustering Energy to Total Network Energy}

The ratio of clustering energy to total network energy is plotted in Figure 5. It can be seen that the clustering process of the proposed IOPEESRA consumes much less energy than that used in M-LEACH and ARPEES. Especially when the number of nodes is large, the energy reduction is more obvious. The reason is that, the probability of a node becoming the cluster head is optimized and each node makes decision to become the cluster head in a distributed fashion.

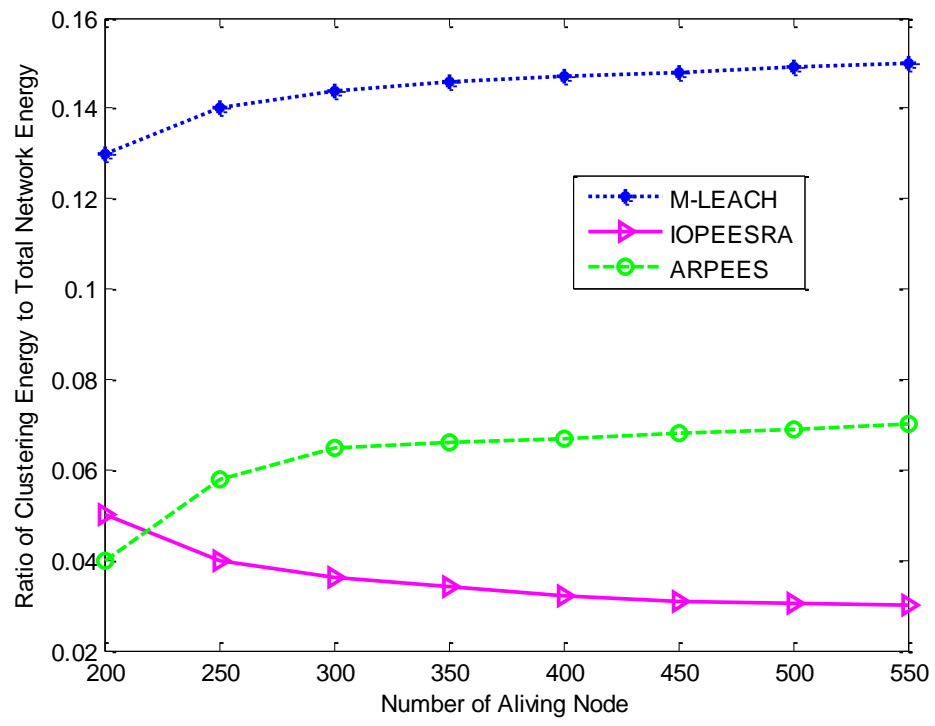

Figure 5. Ratio of Clustering Energy to Total Network Energy 


\section{Results and Discussion}

In this paper, we present an improved optimal path and energy efficiency sensor routing algorithm for athletes' monitoring services. The goal is to pave the way to the realisation of real-time athlete monitoring systems. In our scheme, the basketball court diagram and player default positions are given for the connectivity data collection. Then the radio signal propagation model is set. Next multi-hop routing algorithms for real-time monitoring are analysed. Finally the IOPEESRA algorithm is proposed. Simulation experiments demonstrate the success of the proposed algorithm and its applicability in practical situations. Therefore, it is considered that the IOPEESRA algorithm proposed in this study can be applied to the athletes' monitoring field.

\section{Acknowledgements}

The authors would like to thank the anonymous reviewers for their constructive opinions in improving this paper. The work was supported by the Joint Funds of the National Natural Science Foundation of China and Guangdong Natural Science Foundation ( No.U2012A002D01); Key Projects of National Natural Science Foundation of China (No.U2012A002D01); The Scientific Research Project of Guangzhou City (No: 12C42111582).

\section{References}

[1] K. Koehler, M. K. Parr and H. Geyer, "Serum testosterone and urinary excretion of steroid hormone metabolites after administration of a high-dose zinc supplement", EUROPEAN JOURNAL OF CLINICAL NUTRITION, vol. 41, (2009) January, pp. 65-70.

[2] T. Julie, P. Paul and B. Valerie, "Cardiorespiratory Screening in Elite Endurance Sports Athletes: The Quebec Study”, PHYSICIAN AND SPORTSMEDICINE, vol. 40, (2012) September, pp. 55-65.

[3] W. Julia, "The Royal Flying Doctor Service flies to new heights: the journey of health information management”, HEALTH INFORMATION MANAGEMENT JOURNAL, vol. 38, (2009) December, pp. 5155.

[4] E. S. Rakesh, L. J. Benoit and C. W. Hicks, "Hemodynamic changes as a diagnostic tool in acute heart failure-a pilot study”, AMERICAN JOURNAL OF EMERGENCY MEDICINE, vol. 30, (2012) January, pp. 174-180.

[5] C. Liam and F. Enda, "Human reliability assessment of a critical nursing task in a radiotherapy treatment process", APPLIED ERGONOMICS, vol. 43, (2012) January, pp. 89-97.

[6] O. Spendiff and I. G. Campbell, "Influence of glucose ingestion prior to prolonged exercise on selected responses of wheelchair athletes”, ADAPTED PHYSICAL ACTIVITY QUARTERLY, vol. 20, (2003) January, pp. 80-90.

[7] D. N. Ranalli Denni and D. L. Elderkin, "Oral health issues for adolescent athletes", Dental clinics of North America, vol. 50, (2006) January, pp. 119-137.

[8] V. -A. Javier, P. Lopez-Matencio and F. J. Gonzalez-Castano, "Ambient Intelligence Systems for Personalized Sport Training”, SENSORS, vol. 10, (2010) March, pp. 2359-2385.

[9] K. J. Luella and B. S. Irene, "Eating attitudes and behaviours in elite Canadian athletes with a spinal cord injury", Eating behaviors, vol. 13, (2012) January, pp. 36-41.

[10] S. Manolis, F. Michael and H. David, "Event Detection and Correlation for Network Environments", IEEE JOURNAL ON SELECTED AREAS IN COMMUNICATIONS, vol. 28, (2010) January, pp. 60-69.

[11] B. Mitra, M. Nirvana and P. J. M. Havinga, "Sensing Solutions for Collecting Spatio-Temporal Data for Wildlife Monitoring Applications: A Review”, SENSORS,vol. 13, (2013) May, pp. 6054-6088.

[12] S. Tarik, H. Serkan and I. Serkan, "Some cellular immune components and C-reactive protein monitoring in female handball players during a competitive period", AFRICAN JOURNAL OF MICROBIOLOGY RESEARCH,vol. 5, (2011) January, pp. 173-176.

[13] I. H. Lim, S. Hong and M. S. Choi, "Security Protocols Against Cyber Attacks in the Distribution Automation System”, IEEE TRANSACTIONS ON POWER DELIVERY, vol. 25, (2010) January, pp. 448455.

[14] K. Psannis and Y. Ishibashi, "Efficient Error Resilient Algorithm for H.264/AVC: Mobility Management in Wireless Video Streaming”, Springer Telecommunication Systems Journal, vol. 41, (2009) June, pp. 65-76. 
[15] W. Williams and J. Purnell, "THE STATISTICAL DISTRI BUTION OF EXPECTED NOISE LEVEL OUTPUT FROM COMMONLY AVAILABLE PERSONAL STEREO PLAYERS”, ACOUSTICS AUSTRALIA, vol. 38, (2010) December, pp. 119-122.

[16] E. Lopez-Aguilera, C. Jordi and C. Josep, "Propagation delay influence in IEEE 802.11 outdoor networks", WIRELESS NETWORKS, vol.16, (2010) MAY, pp.1123-1142.

[17] M. T. Herd, T. J. Hall and J. Jingfeng, "IMPROVING THE STATISTICS OF QUANTITATIVE ULTRASOUND TECHNIQUES WITH DEFORMATION COMPOUNDING: AN EXPERIMENTAL STUDY”, ULTRASOUND IN MEDICINE AND BIOLOGY, vol. 37, (2011) December, pp. 2066-2074.

[18] S. Luo, X. Lu and L. Cheng, "TSOIA: An efficient node selection algorithm facing the uncertain process for Internet of Things", JOURNAL OF NETWORK AND COMPUTER APPLICATIONS, vol. 36, (2013) March, pp.738-743.

[19] A. M. Ortiz, T. Olivares and L. Orozco-Barbosa, "Smart Routing Mechanism for Green ZigBee-based Wireless Sensor Networks", 16th IEEE Symposium on Computers and Communications (ISCC), Corfu, GREECE, (2011) JUNE 28-JULY 01.

[20] X. Shuo, D. Ashay and S. Vijay, "Transmission Power Control in Body Area Sensor Networks for Healthcare Monitoring", IEEE JOURNAL ON SELECTED AREAS IN COMMUNICATIONS, vol. 27, (2009) January, pp. 37-48.

\section{Authors}

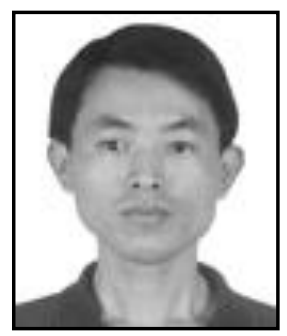

\section{Shi Liang Luo}

Shi Liang Lou was born on October, 1978 in JiangXi, China. He received his M.S degree in automation from Guangdong University of Technology, GuangDong, China, in 2005. Currently he is pursuing Ph.D degree in Control Science and Engineering at Guangdong University of Technology. His research interests include Athlete Monitoring, cyberphysical systems and wireless sensor networks.

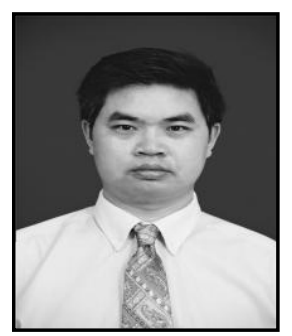

\section{LiangLun Cheng}

Liang Lun Cheng was born on August 22, 1964 in HuBei. He received his M.S and Ph.D degrees from Huazhong University of Science and Technology, HuBei, China in 1992 and Chinese academy of Sciences JiLin, china in 1999 respectively. He is a Prof and doctoral supervisor of Guangdong University of Technology. His research interests include 3G wireless video transmissio, RFID and WSN, IoT and CPS, production equipment and automation of the production process, embedded system, the complex system modeling and its optimization control, software of automation and information, etc.

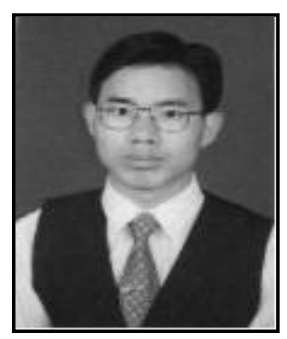

Bin Ren received the Ph.D. degree in control theory and control applications from Guangdong University of Technology China. Currently, he is a researcher at Dongguan University of Technology, China. His major research interests include algorithm design and image processing. 
International Journal of Control and Automation Vol.7, No.4 (2014) 